\title{
BMJ Open Exploring research on the coping strategies of black survivors of homicide victims: a scoping review protocol
}

\author{
Travonne Edwards (1) , Tanya Sharpe (1) , Antonia Bonomo, Notisha Massaquoi
}

To cite: Edwards T, Sharpe T, Bonomo A, et al. Exploring research on the coping strategies of black survivors of homicide victims: a scoping review protocol. BMJ Open 2021;11:e049784. doi:10.1136/ bmjopen-2021-049784

- Prepublication history for this paper is available online. To view these files, please visit the journal online (http://dx.doi org/10.1136/bmjopen-2021 049784).

Received 02 February 2021 Accepted 08 0ctober 2021

Check for updates

(C) Author(s) (or their employer(s)) 2021. Re-use permitted under CC BY-NC. No commercial re-use. See rights and permissions. Published by BMJ.

Factor-Inwentash School of Social Work, University of Toronto, Toronto, Ontario, Canada

Correspondence to Mr Travonne Edwards; Travonne.edwards@mail. utoronto.ca

\section{ABSTRACT}

Introduction Black people are disproportionately impacted by homicide. However, despite this overrepresentation, research is limited relevant to how black individuals, families and communities cope with the chronic traumatic devastation of homicide. This scoping review will provide an amalgamation of the current literature regarding the coping strategies of black survivors of homicide victims to inform future health and social work practice.

Methods and analysis In this scoping review, Arksey and 0'Malley's five-stage framework will be used to succinctly gather and synthesise previous literature and identify gaps in research relevant to black survivors of homicide victims. This method will allow for a focused process of chosen pertinent databases. The seven databases include OVID (MEDLINE and PsycINFO), ProQuest (Sociological Abstracts, Social Services Abstracts, International Bibliography of Social Sciences), EBSCO (Africa Wide, The Cumulative Index to Nursing and Allied Health Literature). The seven databases were chosen for their relevance to the topic of coping with homicide for black individuals, families and communities. All members of the research team will screen the abstracts and full texts of the literature based on the inclusion criteria. The findings will be charted and synthesised using a qualitative thematic analysis.

Ethics and dissemination The articles chosen for this review will be gathered from peer-reviewed journals and scholarly search engines. Due to this research project's nature, ethics approval is not warranted. The results of this scoping review will inform culturally responsive approaches to research, policy and practice for first responders (eg, law enforcement, emergency medical technicians) and providers (eg, mental health clinicians, physicians and faith-based communities) who frequently render services to black survivors of homicide victims. The results will be shared through journal article publications, academic and community conferences, as well as professional training opportunities for practitioners who support Black individuals, families and communities.

\section{INTRODUCTION}

Black people suffer disproportionately from direct and indirect exposure to homicide. ${ }^{1-4}$ For the purpose of this scoping review protocol, the term 'homicide' will be defined as the killing of one person by another. Homicide is a more expansive and common term

\section{Strengths and limitations of this study}

- This study comprehensively synthesises evidence across a range of study designs and methods for the purpose of examining scholarship relevant to the coping strategies of black survivors of homicide victims.

- This scoping review will contribute to the gap in research pertaining to black individuals, families and/ or communities methods of coping and make recommendations for future research.

- This scoping review protocol was developed in consultation with the University of Toronto FactorInwentash Faculty of Social Work librarian.

- This research study is limited to empirical studies published in English within the last 20 years, and will exclude book reviews, dissertations, full books and grey literature.

that refers to non-criminal and criminal acts of murder. ${ }^{5}$ In the USA, black people comprise approximately $13 \%$ of the population, and yet account for $50 \%$ of all homicide victims annually. ${ }^{6}$ In Canada, black people represent $3.5 \%$ of the population, yet encompass $44 \%$ of homicide victims. ${ }^{78}$ Despite the disproportionate prevalence of homicide among black communities in North America, there is limited research exploring their coping strategies following the loss of a loved one. ${ }^{9} 10$ It is estimated that each homicide victim leaves behind 7-10 family, friends, coworkers and neighbours who must survive their violent death. ${ }^{11}$ However, in addition to biological family members, black communities often include chosen persons not related by blood, suggesting that the number of black family members faced with the challenge of coping with the homicide of a loved one may be far greater than what is predicted. ${ }^{12}$

When a loved one is murdered, survivors often find themselves entangled in a complex labyrinth of emotions and reactions. ${ }^{13}$ Experiencing the murder of a loved one interferes with daily functioning, disrupts the ability of survivors to make meaning of the world 
around them and has profound consequences on their overall well-being, productivity and quality of life. ${ }^{14-17} \mathrm{On}$ being informed about the murder, survivors of homicide victims often experience feelings of shock, rage, guilt, helplessness, grief, isolation and dissociation. ${ }^{11} 13$ 18-20 Moreover, scholars have demonstrated that due to the unanticipated nature of homicide, survivors are at greater risk for depression, post-traumatic stress disorder (PTSD), as well as chronic and/or complicated grief. ${ }^{12} 14181921$

The psychological, social and physical effects of traumatic loss are articulated through the practice of grief. ${ }^{22}$ How individuals grieve depends on many factors including the circumstances of the homicide, access and utilisation of medical and mental health services, use and availability of informal support systems (eg, family members and friends), the involvement of the criminal justice system, the nature of the relationship with the deceased, religious and/or cultural beliefs. ${ }^{2023-30}$ Moreover, Sharpe ${ }^{31}$ found that the coping strategies and support systems of black survivors of homicide victims are influenced by racebased social and structural inequities. Black survivors of homicide victims who experience the traumatic murder of a loved one does not simply call for an appraisal of the 'threat' to determine what types of coping strategies are available for use to ensure one's well-being. Rather, black people racially appraise the impact of experiencing homicide through structurally racist systems that place value on the experience, and bring about feelings of stigma, shame, blame and lack of justice.

Connolly and Gordon ${ }^{10}$ conducted a systematic review which explored the effects of homicide on surviving family members. Results indicated that exposure to the homicide of a loved one profoundly impacted the lives of surviving victims psychologically, academically, socially, occupationally, as well as disrupting routine family dynamics. ${ }^{10}$ Additionally, findings indicated that the grieving process was also disrupted and postponed when survivors are interacting with the criminal justice system due to a lack of transparency of institutional processes, lack of sympathy and lengthy investigations. ${ }^{10}$ Lastly, survivors found that fostering supportive networks and spirituality were meaningful coping strategies for managing their grief and building resiliency. Relatedly, Alves-Costa et al. ${ }^{32}$ conducted a systematic review that investigated psychological interventions available and their effectiveness to address survivors of homicide victims mental health symptoms. Findings indicated that cognitivebehavioural therapy, restorative retelling, eye movement desensitisation and reprocessing group therapy were effective in reducing symptoms of PTSD, complicated grief, and depression for survivors of homicide victims.

While these systematic reviews provide insight into the traumatic impact of homicide on survivors and highlight interventions designed to help them cope with their grief, gaps in the posthomicide literature remain. Despite the disproportionate experiences of homicide for black individuals, families and communities, they are seldomly represented in posthomicide research. Granek and
Peleg-Sagy ${ }^{33}$ conducted a systematic review to investigate the representation of African-Americans in the grief literature. Findings indicated that several studies were missing demographic details (eg, race, ethnicity, age, education, socioeconomic status). The absence of sociodemographic data impedes on the contextualisation of grief and validity of findings. In addition, findings revealed that white populations were often used as the normative standard to which black populations are compared. The lack of black participants in research on coping with homicide neglects the disproportionate reality of their experiences as well as fails to identify culturally responsive interventions and strategies designed to support black individuals, families and communities in coping with homicide. Moreover, this void in posthomicide research illustrates an incomplete narrative relevant to the diverse sociocultural experiences and coping strategies of black survivors of homicide victims. ${ }^{34}$

The scarcity of research on black survivors of homicide victims, leaves social workers, medical professionals and practitioners with minimal data to develop culturally responsive evidence-based interventions that support survivors as they grieve the murder of their loved one (s). ${ }^{9}$ In order to address these gaps in research, this scoping review will aim to systematically organise the literature pertaining to the coping strategies of black survivors of homicide victims.

This study will be guided by the following three research questions: (1) What is the impact of experiencing the homicide of a loved one for black individuals, families and communities? (2) How do black individuals, families and communities cope with experiencing the homicide of a loved one? And (3) what strategies and/or interventions have been designed to support black individuals, families and communities coping with homicide? This methodological approach will provide a succinct systematic synthesis of the literature, providing a valuable resource to health practitioners, social workers, researchers, advocates and black survivors of homicide victims.

\section{METHODS AND ANALYSIS}

This scoping review aims to identify and synthesise the current literature on how black survivors of homicide cope with the loss of a loved one. A scoping review is a useful method to understand the scope of the literature on a particular area and to provide clarity regarding what research currently exists, identify gaps and to clarify concepts. ${ }^{35}$ Moreover, scoping reviews can identify understanding how research has been conducted in each discipline or on a topic. ${ }^{35}$ Scoping reviews are helpful for investigations when developing research remains ambiguous and more precise questions can be posed,whereas systematic reviews are generally understood as a research method of synthesis that is used by investigators who seek to identify and extract evidence relevant to a particular topic, and to evaluate the quality of the findings to better inform policy, practice and future research. ${ }^{35} 36 \mathrm{~A}$ 
systematic approach employs explicit methods to reduce bias, which support the production of reliable results that can appropriately inform decision-making processes. ${ }^{35}$

Due to the limited research on black experiences of coping with homicide, a scoping review is an effective methodological approach of generating knowledge in a manner that systematically maps out study findings, strategies used to cope with the experience and interventions used to support black survivors of homicide victims. As such, the authors of this study decided to conduct a scoping review to map the existing research pertaining to the coping strategies of black survivors of homicide victims.

This scoping review protocol uses Arksey and O'Malley's ${ }^{37}$ five-phase scoping review framework that includes: (1) indicating the core research questions; (2) highlighting related research; (3) organising the research that meets the determined inclusion criteria; (4) outlining the findings ${ }^{5}$; analysing and disseminating the final results. Moreover, this study will adhere to the Preferred Reporting Items for Systematic Reviews and Meta-Analyses Extension for Scoping Reviews guidelines (PRISMA-ScR).

\section{Stage 1: outline the research question}

As articulated in our literature review, this research interest emerged from an understanding that despite the disproportionate impact of homicide on Black individuals, families and communities, post-homicide research is often devoid of their experiences. Moreover, existing research that does exist relevant to the impact, coping strategies and approaches designed to assist Black survivors of homicide victims has not been systematically collected and analysed. Across the research, the constructed group "Black" is not utilised consistently ${ }^{33}$; some scholars use a variety of terminologies and some participants identify with different ethnic groups. ${ }^{38}$ This scoping review will also use an array of search terms such as Black, African-American, African, Caribbean, and Afro (eg, Caribbean, Brazilian, etc.) to capture the nuances of the diversity of Blackness within the various vocabulary indexes across the included databases.

The following research questions were developed by the lead (TE) and second author (TS): (1) What is the impact of experiencing the homicide of a loved one for Black individuals, families and communities? (2) How do Black individuals, families and communities cope with experiencing the homicide of a loved one? And (3) what strategies and/or interventions have been designed to support Black individuals, families, and communities coping with homicide?

\section{Stage 2: identifying relevant studies}

This scoping review strategy was developed collaboratively among the authors and reviewed in partnership with the University of Toronto, Factor-Inwentash Faculty of Social Work Librarian. See Box 1 for the draft search strategy in OVID PsycINFO. We engaged in a peer review process to assess the fit of potential databases. The following online

\section{Box 1 Search Strategy Ovid (PsycINF0)}

1. (Black* or "African American*" or Afro* or "African Cultural Group*" or African $\left.{ }^{\star}\right)$.tw.

2. Blacks/

3. African cultural groups/

4. or $/ 1-3$

5. ((Homic ${ }^{*}$ or Murder ${ }^{*}$ or Crime ${ }^{*}$ or Violen*) adj2 (Victim ${ }^{*}$ or Survivor $\left.{ }^{*}\right)$.tw.

6. crime victims/

7. victimisation/

8. exp homicide/

9. survivors/

10. or $/ 5-9$

11. (Copi* or "Social Support"” or Resilien* or Bereave* or Mourn* or Grief* or "Mental Health" or Trauma* or "Post Traumatic Stress Disorder" or PTSD).tw.

12. coping behavior/

13. exp posttraumatic stress disorder/

14. exp bereavement

15. trauma/ or emotional trauma/ or moral injury/ or post-traumatic stress/ or posttraumatic growth/ or traumatic loss/

16. social support/

17. "Resilience (Psychological)"/

18. mental health/

19. or $/ 11-18$

20. 4 and 10 and 19

databases were chosen due their applicability and transdisciplinary perspectives in relation to black homicide victims and their family members and friends surviving their murder. The selected databases consist of: OVID (MEDLINE and PsycINFO), Proquest (Sociological Abstracts, Social Services Abstracts, International Bibliography of Social Sciences), EBSCO (Africa Wide, The Cumulative Index to Nursing and Allied Health Literature). To support the development of the search string terms, the authors visited and considered each database's vocabulary index to assure the search terms were suitable based on the journal's search criteria. The chosen literature will be imported into Covidence software which will aid in the peer-review analysis process helping to ensure the quality and rigour of the scoping review. All chosen articles for this study will follow the inclusion criteria and will be assessed by a peer-review screening of key words, titles and abstracts.

During the title and abstract screening process, literature that articulates the impact of experiencing the murder of a loved one for black survivors of homicide victims, strategies used by black survivors to cope with the homicide of a loved one, interventions or approaches used to support black people who have experienced the homicide of a family member or friend will be selected for the full abstract screening. Despite the historical and disproportionate impact of homicide on black communities, the past two decades have exposed the pervasive nature of homicide, requiring an examination of ways to support black individuals, families and communities cope with such tragedy. Therefore, this scoping review will only 
include scholarly articles published throughout the years $2000-2020$ to focus on contemporary research designed to understand the coping strategies of black survivors of homicide victims.

\section{Stage 3: selecting studies that meet the inclusion criteria}

This scoping review will include empirical studies that use a diverse a array of methods quantitative (eg, administered and self-report survey) and qualitative (e.g., individual and focus group interviews) to examine the coping strategies of black individuals, families and/or communities. There are no geographical or methodological limitations within this review. Manuscripts must meet the following inclusion criteria: (1) include the word black or African (eg, African-American and Afro Caribbean) in the title and abstract; (2) include the word homicide or one of the following terms used synonymously with this construct in the title or abstract (e.g., murder, death, killing, fatality); (3) include the word coping or one of the following synonymous terms in the title and abstract (e.g., manage, survive, healing, resilience) and (4) have an explicit focus on black survivors of homicide and how they cope with this form of tragic death. Strategies and interventions must have a majority sample of black participants to be included in this scoping review. This study will exclude book reviews, dissertations, full books, studies where black survivors of homicide victims are not included in study sample, conceptual articles, nonEnglish articles and grey literature (newspapers, governmental documents and non-peer-reviewed articles).

The screening of the articles will involve a peer review process. Covidence will be used to support efficient screening, article review and charting during this process. Each article will be required to be reviewed and approved by two screeners in order to meet the inclusion criteria of this study. The two first authors will be responsible for resolving all conflicting decisions that may emerge within the review.

In order to establish and assess the quality and agreement rates of our screening process, we will use the fist 100 articles using the inclusion and exclusion criteria identified. For this scoping review protocol, the Kappa score will be used to measure the interagreement rate. This project will achieve a $90 \%$ interagreement prior to independently screening. If this interagreement rate is not achieved from the first 100 articles, we will reconvene to clarify the purpose and criteria of this study. This process will then be repeated with another 100 articles to achieve a $90 \%$ interagreement score.

\section{Stage 4: chart data key results}

Article themes will be charted to present their content as it relates to the research questions. Content will be reviewed and charted under the following headings: authors, year of publication, country where article was published and/or country the research focuses on, name of journal, study method (eg, qualitative, quantitative, mixed methods), sample participants (eg, gender, race/ ethnicity, age), research question categories (impact of homicide, coping strategies, intervention/approaches), recommendations for research, policy and practice.

The extraction of the literature will be facilitated by the first and secondauthor. Any discrepancies that may occur throughout the extraction process will be resolved collaboratively by the authors; if any conflicts remain, we will consult with the University of Toronto, Factor-Inwentash Faculty of Social Work librarian for feedback.

\section{Stage 5: synthesis and report findings}

Findings will be organised and synthesised based on the methodological approach of the study. Quantitative data descriptive statistics, frequencies and variance throughout the data will be reported. Qualitative data will be thematically analysed while the mixed-methods data will follow a blend of both approaches as appropriate. The results of this scoping review will be disseminated as published scholarly material, as well as through poster format at relevant peer-reviewed conferences. It is anticipated that findings will identify and outline the importance of advancing culturally responsive research, policy and practice as pertains to over-represented populations of black survivors of homicide victims.

\section{ETHICS AND DISSEMINATION}

The scoping review study aims to chart out and synthesise the contemporary research on how black individual, families and communitie cope with homicide. The studies used in this scoping review were chosen from popular journals databases and scholarly search engines (eg, ProQuest, OVID, SAGE, Springer). As this research uses non-primary data sources, ethics approval was not required. The findings of this scoping review will be disseminated through academic journals, academic and community conferences. Findings will also be shared with key stakeholders in black communities most impacted by homicide to ensure that the evidence produced is grounded, relevant, accessible and useful.

Twitter Tanya Sharpe @DrTSharpe and Notisha Massaquoi @NotishaMassaqu1

Contributors All the authors made tremendous intellectual contributions to the production of this scoping review protocol. The research questions were created by TE and TS. TE and AB reviewed the vocabulary indexes of all the journal databases in consultation with TS. TE led the consultation with the UofT librarian and the development of the search strategy and protocol, as well as edited the protocol in consultation with NM and the Health Sciences Writing Centre at the University of Toronto. Revisions and improvements to the search strategy were completed in collaboration with the coauthors (TE, TS, AB and NM) for the formal submission for journal review.

Funding This study was funded by the University of Toronto Provost's Office and The Division of University Advancement.

Competing interests None declared.

Patient and public involvement Patients and/or the public were not involved in the design, or conduct, or reporting, or dissemination plans of this research.

Patient consent for publication Not applicable.

Provenance and peer review Not commissioned; externally peer reviewed.

Open access This is an open access article distributed in accordance with the Creative Commons Attribution Non Commercial (CC BY-NC 4.0) license, which 
permits others to distribute, remix, adapt, build upon this work non-commercially, and license their derivative works on different terms, provided the original work is properly cited, appropriate credit is given, any changes made indicated, and the use is non-commercial. See: http://creativecommons.org/licenses/by-nc/4.0/.

\section{ORCID IDs}

Travonne Edwards http://orcid.org/0000-0001-6054-2774

Tanya Sharpe http://orcid.org/0000-0003-4437-1957

Notisha Massaquoi http://orcid.org/0000-0002-9674-4500

\section{REFERENCES}

1 Mobasseri S. Race, place, and crime: how violent crime events affect employment discrimination. Am J Sociol 2019;125:63-104.

2 Jiang T, Webster JL, Robinson A, et al. Emotional responses to unintentional and intentional traumatic injuries among urban black men: a qualitative study. Injury 2018;49:983-9.

3 Rudrow KJ. "I See Death Around the Corner": Black Manhood and Vulnerability in Me Against the World. J Black Stud 2019;50:632-50.

4 Singletary G. Beyond PTSD: black male fragility in the context of trauma. J Aggress Maltreat Trauma 2020;29:517-36.

5 Britannica. The Editors of Encyclopaedia. Homicide. In: Encyclopedia Britannica [Internet, 2020. https://www.britannica.com/topic/ homicide

6 Violence Policy Center. Black Homicide Victimization in the United States: An Analysis of 2016 Homicide Data [Internet], 2019May. Available from. Available: http://vpc.org/studies/blackhomicide19.pdf

7 Maheux H, Do D. Diversity of the black population in Canada: an overview. [Internet]. Ethnicity, Language and Immigration Thematic Series: Statistics Canada, 2019. Available: http://publications.gc.ca/ collections/collection_2019/statcan/89-657-x/89-657-x2019002-eng. pdf [Accessed cited 2021 Jan 14].

8 Moreau G, Jaffray B, Armstrong A. Police-reported crime statistics in Canada, 2019 (report No.: 1209-6393). Staistics Canada, 2020: 1-69.

9 Sharpe TL, Iwamoto DK, Massey JM, et al. The development of a culturally adapted pilot intervention for African American family members of homicide victims: a preliminary report. Violence Vict 2018;33:708-20.

10 Connolly J, Gordon R. Co-victims of homicide. Trauma Violence Abuse 2015;16:494-505.

11 Redmond LM. Surviving: When Someone You Love Was Murdered - A Professional's Guide to Group Grief Therapy for Families and Friends of Murder Victims. Psychological Consultation and Education Services 1989:171.

12 Sharpe TL. Understanding the sociocultural context of coping for African American family members of homicide victims. Trauma Violence Abuse 2015;16:48-59.

13 Rynearson EK, McCreery JM. Bereavement after homicide: a synergism of trauma and loss. Am J Psychiatry 1993;150:258-61.

14 Amick-McMullan A, Kilpatrick DG, Resnick HS. Homicide as a risk factor for PTSD among surviving family members. Behav Modif 1991;15:545-59.

15 Burgess AN. Family reaction to homicide. Am J Orthopsychiatry 1975;45:391-8.

16 Figley CR. Helping traumatized families. Jossey-Bass, 1989.
17 Masters R, Friedman LN, Getzel G. Helping families of homicide victims: a multidimensional approach. J Trauma Stress 1988;1:109-25.

18 Rynearson EK. Bereavement after homicide: a descriptive study. Am J Psychiatry 1984;141:1452-4.

19 Baliko B, Tuck I. Perceptions of survivors of loss by homicide: opportunities for nursing practice. J Psychosoc Nurs Ment Health Serv 2008;46:26-34.

20 Rinear EE. Psychosocial aspects of parental response patterns to the death of a child by homicide. J Trauma Stress 1988;1:305-22.

21 Zinzow HM, Rheingold AA, Byczkiewicz M, et al. Examining posttraumatic stress symptoms in a national sample of homicide survivors: prevalence and comparison to other violence victims. J Trauma Stress 2011;24:743-6.

22 Walter CA, McCoyd JLM. Grief and loss across the lifespan: a biopsychosocial perspective. New York: Springer Pub. Co, 2009: 1-373.

23 Miranda AO, Molina B, MacVane SL. Coping with the murder of a Loved one: counseling survivors of murder victims in groups. The Journal for Specialists in Group Work 2003;28:48-63.

24 Sharpe TL. Sources of support for African-American family members of homicide victims. Journal of Ethnic And Cultural Diversity in Social Work 2008;17:197-216.

25 Spungen D. Homicide: the hidden victims: a guide for professionals. SAGE Publications, 1998.

26 Parkes CM. Psychiatric problems following bereavement by murder or manslaughter. Br J Psychiatry 1993;162:49-54.

27 Holland JM, Neimeyer RA, Boelen PA, et al. The underlying structure of grief: a Taxometric investigation of prolonged and normal reactions to loss. J Psychopathol Behav Assess 2009;31:190-201.

28 Englebrecht C, Mason DT, Adams MJ. The experiences of homicide victims' families with the criminal justice system: an exploratory study. Violence Vict 2014;29:407-21.

29 Boyas J, Sharpe TL. Racial and ethnic determinants of interracial and ethnic trust. J Hum Behav Soc Environ 2010;20:618-36.

30 Park CL, Benore ER. AUTHORS' RESPONSE: "You're Still There": Beliefs in Continued Relationships With the Deceased as Unique Religious Beliefs That May Influence Coping Adjustment. Int $J$ Psychol Relig 2004;14:37-46.

31 Sharpe TL. Understanding the sociocultural context of coping for African American family members of homicide victims: a conceptual model. Trauma Violence Abuse 2015;16:48-59.

32 Alves-Costa F, Hamilton-Giachritsis C, Christie H, et al. Psychological interventions for individuals bereaved by homicide: a systematic review. Trauma Violence Abuse 2021;22:793-803.

33 Granek L, Peleg-Sagy T. Representations of African Americans in the grief and mourning literature from 1998 to 2014: a systematic review. Death Stud 2015;39:605-32.

34 Waters MC. Black identities. Russel Sage Foundation, 1999.

35 Munn Z, Peters MDJ, Stern C, et al. Systematic review or scoping review? guidance for authors when choosing between a systematic or scoping review approach. BMC Med Res Methodol 2018;18:143.

36 Aromataris E, Pearson A. The systematic review: an overview. Am J Nurs 2014;114:53-8.

37 Arksey H, O'Malley L. Scoping studies: towards a methodological framework. Int J Soc Res Methodol 2005;8:19-32.

38 Edwards T, Hussain A, Sato C. Exploring racial disproportionalities and disparities for black families involved with the child welfare system. Social Science Protocols 2020;10. 